\title{
Characterization of a decapacitation factor associated with epididymal mouse spermatozoa
}

\author{
Lynn R. Fraser, R. A. P. Harrison* and Jane E. Herod \\ Anatomy and Human Biology, Biomedical Sciences Division, King's College London, Strand, \\ London WC2R 2LS, UK, and *Department of Molecular Embryology, \\ AFRC Institute of Animal Physiology and Genetics Research, Babraham, \\ Cambridge CB2 4AT, UK
}

\begin{abstract}
Summary. Earlier studies demonstrated that epididymal mouse spermatozoa have a surface-associated factor which inhibits fertilizing ability in a reversible manner. The factor can be removed from uncapacitated spermatozoa by gentle centrifugation, resulting in immediately highly fertile gametes, and it can be added back to capacitated spermatozoa, resulting in poorly fertile cells in which the acrosome reaction has been blocked. Using such inhibition of in-vitro fertilizing ability as an assay, we have carried out experiments to characterize the factor. It appears to be an anionic polypeptide with $M_{\mathrm{r}}$ of approximately 40000 (according to its behaviour on gel filtration). It is stable to heating at $100^{\circ} \mathrm{C}$ for $15 \mathrm{~min}$ and is not destroyed by proteases at $\mathrm{pH} 8.0$, yet inhibitory activity decreases during sperm incubation in capacitating conditions and is also destroyed in partially purified preparations by endogenous enzyme action during incubation at $\mathrm{pH} 5 \cdot 0$. Activity is not adsorbed to either concanavalin A-agarose or wheatgerm agglutinin-agarose, suggesting that terminal mannose and $\mathrm{N}$-acetylglucosamine residues are not abundant. The factor causes rapid changes in the patterns of chlortetracycline fluorescence seen on sperm heads, a parameter used to assess the capacitated state. Removal of the factor from uncapacitated cells results in a shift to a predominance of capacitated patterns, while the addition of crude or partially purified factor to capacitated cells inhibits the acrosome reaction and causes a shift to the uncapacitated pattern in acrosome-intact spermatozoa. The factor therefore behaves as a decapacitation factor. However, it appears to differ from other characterized decapacitation factors in terms both of molecular size and of abundance of mannose and $\mathrm{N}$-acetylglucosamine residues.
\end{abstract}

Keywords: decapacitation factor; capacitation; acrosome reaction; in-vitro fertilization; mouse spermatozoa

\section{Introduction}

At the time of release from the male reproductive tract, mammalian spermatozoa cannot fertilize eggs immediately but acquire such capability during a species-dependent period of residence in the female tract (Austin, 1951; Chang, 1951). The modifications of the spermatozoon that bring about this functional transformation are termed collectively 'capacitation'; they render the cell able to undergo the acrosome reaction in response to egg-associated factors, and then to penetrate the zona pellucida and fuse with the egg (Yanagimachi, 1988). The observation by Chang (1957) that the addition of seminal plasma to capacitated rabbit spermatozoa caused a reversible inhibition of fertilizing ability, and the later demonstration by Bedford \& Chang (1962) that ultracentrifugation of rabbit seminal plasma sedimented this 'decapacitation factor', initiated studies that have led to the current concept that capacitation involves removal of coating macromolecules from the 
spermatozoon. Decapacitation, the reversal of capacitation, is brought about by re-application (and hence re-adsorption) of these coating substances to the sperm surface.

Since those early studies, numerous decapacitation factors have been detected in a variety of species; the factors, typically proteins or glycoproteins, have been discussed in detail by Oliphant $e t$ al. (1985). The best characterized are an "acrosome-stabilizing factor" isolated from rabbit seminal plasma (Eng \& Oliphant, 1978) and an "antifertility factor" from human seminal plasma (Reddy $e t$ al., 1979). Both of these appear to be large glycoproteins composed of dissimilar subunits (Audhya et al., 1987; Wilson \& Oliphant, 1987). As suggested by its name, acrosome-stabilizing factor blocks fertilization by inhibiting the acrosome reaction (Eng \& Oliphant, 1978), whereas current evidence indicates that the antifertility factor from human seminal plasma acts by a different mechanism, as yet unknown (Reddy et al., 1982).

Previous work (Fraser, 1984) has shown that epididymal mouse spermatozoa have a surfaceassociated component that prevents penetration of the zona pellucida, apparently through inhibition of the acrosome reaction. The removal of the component, which can be accomplished by gentle centrifugation and resuspension of the spermatozoa in fresh medium, rapidly transforms a poorly fertile suspension into a highly fertile one. Conversely, the addition of the component to capacitated suspensions inhibits their fertilizing ability in a temporary manner: given sufficient time, suspensions become able to fertilize rapidly again. The epididymal component thus appears to behave in general terms as a decapacitation factor. Although other macromolecules may be added to the sperm surface at ejaculation, these latter components do not detectably alter the kinetics of capacitation in vitro: ejaculated spermatozoa recovered from uterine horns shortly after mating and placed into modified Tyrode's medium become capable of rapid fertilization at the same rate as do epididymal spermatozoa (Fraser, 1983b). The aim of the study reported below was to isolate and characterize the inhibitory epididymal component and to investigate further its mode of action.

A preliminary account of this work has been published in abstract form (Fraser et al., 1989).

\section{Materials and Methods}

The general materials and methods used for investigating in-vitro fertilization in mice have been described in detail in several previous publications (Fraser, 1983a, b, 1984) and will be given below in outline only.

Preparation of crude inhibitor. Epididymal sperm suspensions were prepared by releasing the contents of each cauda epididymidis from mature male TO mice into a modified Tyrode's medium, lacking pyruvate and lactate but containing glucose, which was supplemented with $0.3 \mathrm{mg}$ bovine serum albumin (BSA; crystalline, from Sigma Chemical Co., Poole, Dorset, UK) per ml; typically, 4 males were used to provide $4 \mathrm{ml}$ suspension. The low level of BSA in the medium as compared with that used for in-vitro fertilization was to facilitate subsequent characterization of the factor.

After $5 \mathrm{~min}$ to allow dispersal of spermatozoa, the suspensions were transferred to small, sterile plastic Bijou containers, gassed with $5 \% \mathrm{CO}_{2}-5 \% \mathrm{O}_{2}-90 \% \mathrm{~N}_{2}$, capped, and then incubated at $37^{\circ} \mathrm{C}$. Liquid paraffin was not used because it interfered with subsequent chromatography. After 25-30 min, suspensions were centrifuged at $11600 \boldsymbol{g}_{\max }$ for $4 \mathrm{~min}$; the epididymal supernatants containing the inhibitor were removed, frozen in liquid $\mathrm{N}_{2}$, lyophilized overnight and stored at $-20^{\circ} \mathrm{C}$.

Partial purification of inhibitor by gel filtration. Lyophilized supernatants were redissolved in $1.2 \mathrm{ml}$ water and centrifuged at $10000 g_{\max }$ for $4 \mathrm{~min}$ at $2^{\circ} \mathrm{C}$. The supernatant was then subjected to gel filtration at $2^{\circ} \mathrm{C}$ on Ultrogel AcA44 (LKB Instruments Ltd, South Croydon, Surrey, UK). The column $\left(0.8 \mathrm{~cm}^{2} \times 60 \mathrm{~cm}\right)$ had been freshly equilibrated with $0.4 \%(\mathrm{w} / \mathrm{v})$ ammonium bicarbonate $(51 \mathrm{~mm}$; approximate $\mathrm{pH} 8.0)$; it was eluted with the same medium at $4 \mathrm{ml} / \mathrm{h}$, and $1.5 \mathrm{ml}$ fractions were collected. The absorbance of the eluted fractions was measured at $280 \mathrm{~nm}$; according to the elution profile, the fractions following the BSA peak and including the maximum of the next absorbance peak were pooled (fraction B according to Fig. 1; see also Fig. 2a and 'Experimental and Results' section relating to gel filtration). The 5 fractions emerging from the column immediately before the first rise in absorbance ('pre-protein' eluate) were also pooled and used for preparing 'blank' samples.

Electrophoresis. Samples of individual fractions from the gel filtration runs or of treated preparations of partially purified inhibitor were subjected to SDS gel electrophoresis, essentially according to Harrison \& Gaunt (1988), using $0.5 \mathrm{~mm}$-thick gradient gels $(5-17 \% \mathrm{w} / \mathrm{v})$. Afterwards, the gels were fixed and stained for protein by a 'silver' method (Morrisey, 1981). 
Preparation of treated inhibitor samples for bioassay. Treated samples were dialysed thoroughly against 100 volumes of freshly prepared $0 \cdot 16 \%(\mathrm{w} / \mathrm{v})$ ammonium bicarbonate $(20 \mathrm{mM})$, using a perturbing roller system (Feinberg, 1976); 5 changes of at least $4 \mathrm{~h}$ each were made. To reduce losses of inhibitor activity, the dialysis tubing (Visking " $8 /$ 32"; The Scientific Instrument Centre, Eastleigh, Hants, UK) was pretreated with sodium carbonate and ethanol according to Richmond et al. (1985). The dialysed samples were then lyophilized for at least $60 \mathrm{~h}$ (to ensure complete removal of ammonium bicarbonate residues).

Bioassay of inhibitor. Sperm suspensions were prepared by releasing the contents of each cauda epididymidis from each of 2 mature TO males into $2 \mathrm{ml}$ modified Tyrode's medium containing BSA at $4 \mathrm{mg} / \mathrm{ml}$. After 5 min to allow spermatozoa to disperse, the suspension was removed and incubated at $37^{\circ} \mathrm{C}$ under autoclaved liquid paraffin in an atmosphere of $5 \% \mathrm{CO}_{2}-5 \% \mathrm{O}_{2}-90 \% \mathrm{~N}_{2}$ for a further $120 \mathrm{~min}$ to ensure complete capacitation (Fraser, 1983a). The lyophilized samples to be assayed were reconstituted in $0.4 \mathrm{ml}$ medium and used to dilute aliquants of the capacitated sperm suspensions. To optimize detection of inhibitor without compromising fertilization by control suspensions, the initial sperm suspensions were diluted approximately $1: 1$ with fresh medium and then a further 10 -fold in the reconstituted samples or control medium; final sperm concentrations were approximately $1 \times 10^{6} \mathrm{cells} / \mathrm{ml}$.

Unfertilized eggs were obtained from mature female TO mice that had been induced to ovulate with i.p. injections of PMSG and hCG. At $14 \mathrm{~h}$ after hCG injection, cumulus-intact eggs were released directly into $400-\mu \mathrm{l}$ droplets of the various diluted sperm suspensions, under liquid paraffin in $30-\mathrm{mm}$ plastic tissue culture dishes, and incubated at $37^{\circ} \mathrm{C}$ in an atmosphere of $5 \% \mathrm{CO}_{2}-5 \% \mathrm{O}_{2}-90 \% \mathrm{~N}_{2}$. After $65 \mathrm{~min}$, the eggs were transferred to droplets of fresh medium and fixed $10 \mathrm{~min}$ later ( $75 \mathrm{~min}$ after mixing of gametes) by flooding the dishes with neutral buffered formalin. The eggs were then stained, mounted and assessed for fertilization. Eggs were considered to be fertilized if they had resumed the second meiotic division and contained a decondensing sperm head.

Chlortetracycline fuorescence assay. A modification of the method described by Ward \& Storey (1984) was used. A droplet $(5 \mu \mathrm{l})$ of sperm suspension was mixed on a clean, warm slide with $5 \mu \mathrm{l} 750 \mu \mathrm{M}$-chlortetracycline hydrochloride (CTC; Sigma) in $130 \mathrm{~mm}-\mathrm{NaCl}, 5 \mathrm{~mm} \cdot$ cysteine, $20 \mathrm{~mm}$-Tris $\mathrm{HCl}$ (final pH 7.8); the CTC was freshly dissolved and the solution kept on ice, shielded from light. After $30 \mathrm{sec}, 0.2 \mu \mathrm{l}$ of $12.5 \%(\mathrm{w} / \mathrm{v})$ paraformaldehyde in $0.5 \mathrm{M}-\mathrm{Tris}-\mathrm{HCl}$ buffer (final $\mathrm{pH} 7.4$ ) was added. Finally, one drop of 0.22 $\mathrm{M}$-1,4-diazabicyclo[2.2.2]octane (Sigma) in glycerol was mixed in to retard fading of fluorescence. A coverslip was added and the slide was compressed between tissues to remove excess liquid, thereby orientating the sperm heads for assessment. Spermatozoa were observed on an Olympus BHS microscope equipped with phase-contrast and epifluorescence optics. The $\mathrm{Hg}$ excitation beam was passed through a $405-\mathrm{nm}$ band-pass filter and CTC fluorescence emission was observed through a DM 455 dichroic mirror.

In each sample, 100 sperm heads were each classified as having 1 of 3 staining patterns: ' $F$ ', with uniform fluorescence over the entire head (characteristic of acrosome-intact uncapacitated spermatozoa); ' $B$ ', with a fluorescencefree dark band in the postacrosomal region (characteristic of acrosome-intact capacitated spermatozoa); 'AR', with fluorescence absent from the head (characteristic of acrosome-reacted spermatozoa). The presence or absence of the acrosomal cap was verified in these samples by using phase-contrast illumination (Fraser, 1983b).

Analysis of data. Data obtained during purification and characterization of the inhibitor were not subjected to statistical analysis. These investigations aimed to detect the relative presence or absence of inhibitor activity in the bioassay, rather than to quantify it precisely. During characterization, the inclusion of 'blank' samples (prepared from 'pre-protein' eluate from the gel filtration column) served as internal standards for comparison.

The CTC assessments involving treatment with crude inhibitor, however, were quantitative in nature. These data were analysed using Cochran's modification of the $2 \times 2$ contingency tables which allows comparison within and between replicates (Snedecor \& Cochran, 1967).

\section{Experimental Details and Results}

\section{Ion-exchange chromatography}

In the first series of experiments, lyophilized samples were desalted into $10 \mathrm{~mm}$-phosphate pH 8.0 by passage through Sephadex G-25 (Pharmacia Ltd, Milton Keynes, Bucks, UK) and then applied to a previously equilibrated column $\left(0.8 \mathrm{~cm}^{2} \times 6.5 \mathrm{~cm}\right)$ of DEAE-Sepharose CL-6B (Sigma). Material which did not adsorb to the DEAE-Sepharose column was adjusted to $\mathrm{pH} 6.0$ and applied to a similar column of CM-Sepharose CL-6B (Sigma) previously equilibrated with $10 \mathrm{~mm}$-phosphate pH 6.0. Each column was then eluted with a 'step' of $1 \mathrm{M}-\mathrm{NaCl}$ in suitably buffered $10 \mathrm{~mm}$-phosphate. Both eluates, as well as the material which did not adsorb to either column, were dialysed against ammonium bicarbonate, lyophilized and then subjected to bioassay. The results (Table $1 \mathrm{a} ; n=2$ replicates) indicated that only material adhering to DEAE-Sepharose possessed inhibitory activity.

In the second series, desalted samples were applied initially to $\mathrm{CM}-$ Sepharose at $\mathrm{pH} 6 \cdot 0$, and the non-adsorbed material was then applied (after adjustmentd to pH 8:0) to DEA Eem Sepharose at 10:10:06AM 
$\mathrm{pH} 8 \cdot 0$. The $\mathrm{CM}-$ Sepharose was eluted with a step of $1 \mathrm{M}-\mathrm{NaCl}$ as before, but the DEAE-Sepharose was eluted with a gradient of $0-2 \mathrm{M}-\mathrm{NaCl}$ in $10 \mathrm{~mm}$-phosphate $\mathrm{pH} 8 \cdot 0$. Eluates were monitored for absorbance at $280 \mathrm{~nm}$. All the material from CM-Sepharose was pooled together as a single fraction, whereas material from DEAE-Sepharose was divided into 3 fractions: that eluting before the main peak of absorbance (assumed to be BSA), that eluting with BSA, and that eluting after BSA. Material that did not adsorb to either column was discarded. Bioassays (Table $1 b ; n=2$ ) indicated that the inhibitory activity was largely in the fraction eluted from the DEAE-Sepharose column after BSA. From these experiments we concluded that the inhibitor is anionic with a high affinity for DEAE-Sepharose.

Table 1. Behaviour of inhibitor on ion-exchange chromatography

\begin{tabular}{lccc}
\hline & \multicolumn{3}{c}{ Eggs fertilized } \\
\cline { 2 - 4 } Sample & No. & $\%$ & (Range, \%) \\
\hline (a) Control (no inhibitor) & $37 / 45$ & $82 \cdot 2$ & $(70-96)$ \\
$\quad$ Bound to DEAE-Sepharose & $1 / 33$ & $3 \cdot 0$ & $(0-6)$ \\
Bound to CM-Sepharose & $54 / 63$ & $85 \cdot 7$ & $(78-96)$ \\
Not bound to either & $21 / 23$ & $91 \cdot 3$ & $(76-100)$ \\
(b) Control & $29 / 29$ & 100 & - \\
Bound to CM-Sepharose & $29 / 41$ & $70 \cdot 7$ & $(40-100)$ \\
Bound to DEAE-Sepharose & & & \\
$\quad$ Eluting before BSA & $42 / 43$ & $97 \cdot 7$ & $(96-100)$ \\
$\quad$ Eluting with BSA & $28 / 33$ & 84.8 & $(78-88)$ \\
$\quad$ Eluting after BSA & $16 / 36$ & $44 \cdot 4$ & $(41-50)$ \\
\hline
\end{tabular}

See text for experimental details. Values are for 2 replicates; each replicate involved material from 8 caudae.

Attempts to develop the use of anion-exchange chromatography to isolate the inhibitor were unsuccessful (data not presented). Bioassay results were inconsistent and losses of activity high; moreover, the positive data we obtained implied that the inhibitor had anionic properties too similar to BSA to allow a ready separation. These studies were therefore not pursued further.

\section{Gel filtration}

Lyophilized samples were applied to columns of Ultrogel AcA44 as described in the 'Materials and Methods'. The absorbance profiles of the eluates showed typically 3 identifiable peaks of macromolecular material, the second of these corresponding to BSA (Fig. 1). Initially, we assayed the material present in each of these peaks plus that in the trough following Peak 3 (Fig. 1, fractionation scheme $a$ ). Results (Table $2 \mathrm{a} ; n=5$ ) indicated that, while no inhibitory activity was associated with either Peak 1 (fraction i) or the trough following Peak 3 (fraction iv), it was present in Peaks 2 (fraction ii) and 3 (fraction iii). In an attempt to localize more closely the position of the inhibitor, subsequent eluates were divided into 3 fractions: A, front half of Peak 2; B, back half of Peak 2 plus trough plus front half of Peak 3; C, back half of Peak 3 (Fig. 1, fractionation scheme $b$ ). Results (Table $2 \mathrm{~b} ; n=5$ ) indicated that the inhibitory activity was most consistently associated with fraction B. As electrophoretic analysis revealed that the material associated with Peak 3 displayed molecular masses of about 27000 or less (Fig. 1), the inhibitor's elution position from Ultrogel AcA44, between Peak 3 and BSA, suggests that it has a molecular mass of about 40000 . Further experiments to characterize the inhibitor utilized preparations of fraction $\mathbf{B}$ as partially purified inhibitor (see 'Materials and Methods'); a representative electropherogram of such a preparation is shown in Fig. 2(a). 
Table 2. Behaviour of inhibitor subjected to gel filtration on Ultrogel AcA44

\begin{tabular}{llcc}
\hline & \multicolumn{3}{c}{ Eggs fertilized } \\
\cline { 2 - 4 } Sample & No. & $\%$ & (Range, \%) \\
\hline (a) Fraction i & $95 / 109$ & $87 \cdot 2$ & $(76-100)$ \\
Fraction ii & $40 / 109$ & $36 \cdot 7$ & $(29-53)$ \\
Fraction iii & $65 / 131$ & $49 \cdot 6$ & $(30-69)$ \\
Fraction iv & $70 / 83$ & $84 \cdot 3$ & $(56-100)$ \\
Control (no & & & \\
$\quad$ inhibitor) & $85 / 99$ & $85 \cdot 9$ & $(75-100)$ \\
(b) Fraction A & $83 / 132$ & $62 \cdot 9$ & $(5-88)$ \\
Fraction B & $62 / 203$ & $30 \cdot 5$ & $(6-71)$ \\
$\quad$ Fraction C & $76 / 123$ & $61 \cdot 8$ & $(36-100)$ \\
Control & $62 / 86$ & $72 \cdot 1$ & $(60-100)$ \\
\hline
\end{tabular}

In (a), eluted fractions were pooled according to scheme $a$ in Fig. $1(n=5)$; in (b), scheme $b$ in Fig. 1 was used $(n=$ 5). Material from 4 caudae was chromatographed in each replicate.

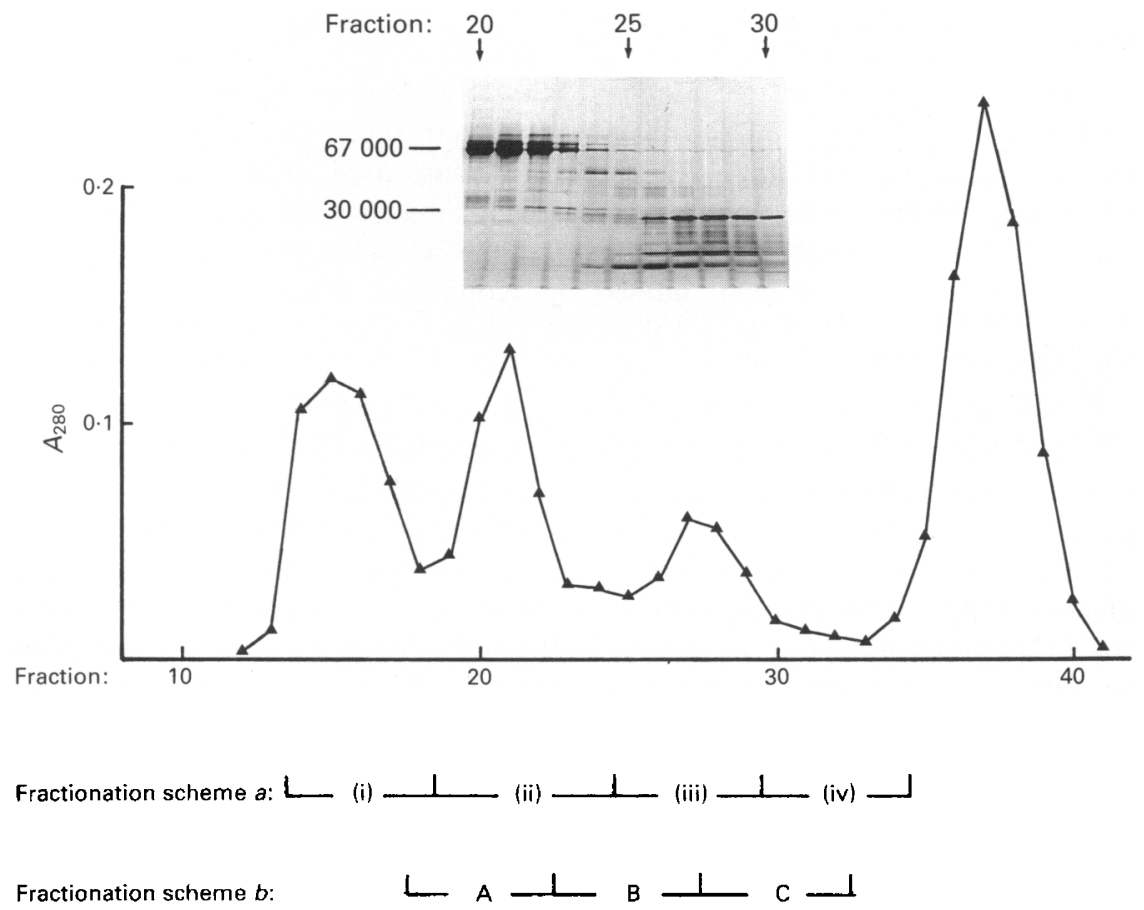

Fig. 1. Gel filtration profile of supernatant from a mouse epididymal sperm suspension. Chromatography was performed on Ultrogel AcA44 as described in the 'Materials and Methods'. Samples from fractions 20-30 were subjected to SDS gel electrophoresis in the reduced state; note the presence of BSA (molecular mass 67 000) in Peak 2 (fractions 19-22). Fractionation scheme $a$ indicates the fraction pools used for the inhibitor assays in Table 2(a), while fractionation scheme $b$ indicates those used for the assays in Table 2(b). Pool B (fractions 23-27) is representative of the preparations of partially purified inhibitor used in the characterization studies. 


\section{Effect of heat treatment}

Partially purified inhibitor was divided into 2 aliquots, one of which was heated at $100^{\circ} \mathrm{C}$ for 15 min while the other was kept in ice. Suitable 'blanks', some including protein (e.g. fraction i, Table 2), were also prepared (both heated and unheated). Bioassays (Table 3;n=5) indicated that inhibitory activity was still present in the preparation after heat treatment. In contrast, heated 'blanks' did not inhibit fertilization, a demonstration that the result with the experimental material was not an artefact generated by boiling material in ammonium bicarbonate.

Table 3. Effect of heating partially purified inhibitor

\begin{tabular}{lrcc}
\hline & \multicolumn{3}{c}{ Eggs fertilized } \\
\cline { 2 - 4 } Sample & \multicolumn{1}{c}{ No. } & $\%$ & (Range, \%) \\
\hline Unheated inhibitor & $65 / 324$ & $20 \cdot 1$ & $(0-60)$ \\
Heated inhibitor & $61 / 315$ & $19 \cdot 4$ & $(7-52)$ \\
Assorted 'blanks' & $229 / 302$ & $75 \cdot 8$ & $(59-84)$ \\
Control (no inhibitor) & $199 / 232$ & $85 \cdot 7$ & $(59-100)$ \\
\hline
\end{tabular}

Values are for 5 replicates; each inhibitor sample contained the material from 4 caudae.

\section{Effect of proteinase treatment at $\mathrm{pH} \mathrm{8.0}$}

Samples of partially purified inhibitor, in $51 \mathrm{~mm}$-ammonium bicarbonate (approximately $\mathrm{pH} 8.0$ ), were treated for $45 \mathrm{~min}$ at $37^{\circ} \mathrm{C}$ with either trypsin (product $\mathrm{T} 8128$ from Sigma) or pronase E (product P6911 from Sigma); the amounts of proteinase used were sufficient to cause gross degradation, within $15 \mathrm{~min}$, of a quantity of azocasein similar to the quantity of protein in the inhibitor samples. At the end of the incubation period, the samples were heated at $100^{\circ} \mathrm{C}$ for $15 \mathrm{~min}$ to inactivate the proteinases, and then dialysed and lyophilized. Samples without proteinase and 'blanks' ('pre-protein' eluate from the gel filtration column, with and without added proteinase) were similarly treated.

Bioassays indicated that neither proteolytic enzyme had any effect on inhibitory activity (Table $4 ; n=4)$. Enzyme-treated blanks were similar to untreated blanks, demonstrating that the presence of heat-inactivated proteinases did not detectably modify sperm fertilizing activity. Electrophoretic analysis (data not shown) indicated that in all experiments proteolytic digestion had taken place, each enzyme engendering specific alterations in some (though not all) of the constituent components of the partially purified inhibitor preparation; no endogenous proteolysis could be detected (see also Fig. 2b). From these results we conclude that the inhibitory activity is not sensitive to proteinase action at $\mathrm{pH} 8 \cdot 0$.

\section{Effect of guanidine hydrochloride and dithiothreitol}

Proteins are susceptible both to disruptors of non-covalent interactions and to reducing agents that disturb the disulphide cross-linking of their polypeptide chains. The methodology was based on that described by Perham (1978). Aliquants of partially purified inhibitor (in $51 \mathrm{~mm}$-ammonium bicarbonate) were treated for $30 \mathrm{~min}$ at $37^{\circ} \mathrm{C}$ with various levels of guanidine hydrochloride, in the presence or absence of $3 \mathrm{~mm}$-dithiothreitol (final concentration); other aliquants were treated with dithiothreitol alone or were simply incubated at $37^{\circ} \mathrm{C}$. Various 'blanks' were similarly treated. At the end of the incubation period, all samples were placed on ice, and, to those samples that had received dithiothreitol, excess iodoacetamide was added to block all exposed thiol groups. After a further $5 \mathrm{~min}$, sufficient 2-mercaptoethanol was added to these samples to block unreacted iodoacetamide. Then all samples were dialysed, lyophilized, and subjected to bioassay 
Table 4. Effect on partially purified inhibitor of proteinase treatment at $\mathrm{pH} 8.0$

\begin{tabular}{lrrc}
\hline & \multicolumn{3}{c}{ Eggs fertilized } \\
\cline { 2 - 4 } Sample & \multicolumn{1}{c}{ No. } & $\%$ & (Range, \%) \\
\hline Untreated inhibitor & $15 / 112$ & $13 \cdot 4$ & $(0-28)$ \\
Inhibitor (heated only) & $2 / 130$ & $9 \cdot 2$ & $(0-18)$ \\
Inhibitor + trypsin & $41 / 173$ & $23 \cdot 7$ & $(0-71)$ \\
Inhibitor + pronase E & $4 / 161$ & $2 \cdot 5$ & $(0-8)$ \\
Assorted 'blanks' & $275 / 385$ & $71 \cdot 4$ & $(25-90)$ \\
Control (no inhibitor) & $108 / 152$ & $71 \cdot 1$ & $(44-97)$ \\
\hline
\end{tabular}

See text for experimental details. Values are for 4 replicates; each inhibitor sample contained the material from 2 caudae.

Some variation in results was observed, partly because there was a tendency for treated 'blanks', as well as the samples of inhibitor, to interfere with sperm-egg fusion and therefore to block fertilization. However, careful observation of the unfixed eggs showed that in many cases spermatozoa had penetrated the zona pellucida. Because the active inhibitor specifically blocks this process (Fraser, 1984), we decided that activity was absent if sperm penetration of the zona pellucida had taken place, regardless of whether actual fertilization had occurred. In this way, it was possible to conclude that the inhibitory activity was sensitive to dithiothreitol in the presence of guanidine hydrochloride (Table $5 ; n=4$ ), and therefore that almost certainly the activity resided in a protein of some kind.

Table 5. Effect on partially purified inhibitor of dithiothreitol (DTT) and guanidine hydrochloride $(\mathrm{GuHCl})$

\begin{tabular}{lccc}
\hline & \multicolumn{3}{c}{ Eggs fertilized } \\
\cline { 2 - 4 } Sample & No. & $\%$ & (Range, \%) \\
\hline Untreated inhibitor & $23 / 132$ & $17 \cdot 4$ & $(0-45)$ \\
Inhibitor + GuHCl & $19 / 85$ & $22 \cdot 4$ & $(0-55)$ \\
Inhibitor + GuHCl + DTT & $91 / 159^{*}$ & $57 \cdot 2$ & $(18-80)$ \\
Inhibitor + DTT & $72 / 148$ & $48 \cdot 6$ & $(0-86)$ \\
Assorted 'blanks' & $178 / 265^{*}$ & $67 \cdot 2$ & $(0-95)$ \\
Control (no inhibitor) & $96 / 115$ & $83 \cdot 5$ & $(67-100)$ \\
\hline
\end{tabular}

${ }^{*}$ Perivitelline spermatozoa present in the majority of unfertilized eggs (see text); numerator given represents only fertilized eggs with decondensing sperm heads. Values are for 4 replicates; each inhibitor sample contained the material from 2 caudae.

\section{Chromatography on immobilized lectins}

The stability of the inhibitor to heat and its resistance to proteolytic attack at $\mathrm{pH} 8$ suggested that its active moiety might involve carbohydrate residues. Accordingly, its affinity for lectins was investigated.

Partially purified inhibitor was applied to concanavalin A-agarose (ConA-ag; BDH, Poole, Dorset, UK) or to wheat-germ agglutinin-agarose (WGA-ag; Pharmacia) at pH 7.5. Each immobilized lectin was in the form of a $1-\mathrm{ml}$ column, and was pre-washed with Hepes-buffered $0.5 \mathrm{M}-\mathrm{NaCl}$ and $\alpha$-methylmannoside (ConA-ag) or $N$-acetylglucosamine (WGA-ag), before equilibration with $0 \cdot 12 \mathrm{M}-\mathrm{NaCl} / 20 \mathrm{~mm}-\mathrm{Hepes} \mathrm{pH} 7 \cdot 5$. Samples were cycled through the columns three 
times to ensure interaction with the lectin, and the columns were then washed thoroughly with Hepes-buffered $0.5 \mathrm{M}-\mathrm{NaCl}$; the washings were pooled with the cycled samples, to constitute the 'unbound' fractions. The ConA-ag was then eluted with $0.3 \mathrm{M}-\alpha$-methylmannoside in Hepesbuffered saline at $\mathrm{pH} 7 \cdot 5$, while the WGA-ag was eluted with $0.3 \mathrm{M}-N$-acetylglucosamine; the eluates constituted the 'bound' fractions. A 'blank' was constructed of equivalent quantities of ammonium bicarbonate, Hepes buffer, $\mathrm{NaCl}, N$-acetylglucosamine and $\alpha$-methylmannoside.

Bioassays indicated that inhibitory material did not bind detectably to either column (see Table 6 for ConA-ag A data, $n=2$; WGA-ag data not presented, $n=1$ ). It was concluded that the inhibitor did not contain abundant terminal residues of mannose and $\mathrm{N}$-acetylglucosamine.

Table 6. Chromatography of partially purified inhibitor on concanavalin A-agarose

\begin{tabular}{lrcc}
\hline & \multicolumn{3}{c}{ Eggs fertilized } \\
\cline { 2 - 4 } Sample & \multicolumn{1}{c}{ No. } & $\%$ & (Range, \%) \\
\hline Untreated inhibitor & $24 / 57$ & $42 \cdot 1$ & $(7-54)$ \\
Bound to ConA-ag & $28 / 42$ & $66 \cdot 7$ & $(50-71)$ \\
Not bound to ConA-ag & $5 / 44$ & $11 \cdot 3$ & $(0-17)$ \\
Assorted 'blanks' & $48 / 54$ & $88 \cdot 8$ & $(60-100)$ \\
Control (no inhibitor) & $45 / 45$ & 100 & - \\
\hline
\end{tabular}

Values are for 2 replicates; each inhibitor sample contained the material from 4 caudae.

\section{Incubation at $\mathbf{p H} 5 \cdot 0$}

The original intention was to treat the inhibitor.with commercially available exoglycosidases to investigate further the possibility that carbohydrate moieties were involved in inhibitor action. As such glycosidases have $\mathrm{pH}$ optima near $\mathrm{pH} 5$, it was deemed necessary to carry out treatment at this $\mathrm{pH}$ to maximize the likelihood of obtaining positive results. However, preliminary experiments indicated that 'control' incubation at $\mathrm{pH} 5$ resulted in loss of inhibitor activity, so this sensitivity was investigated further.

Two aliquants of partially purified inhibitor were adjusted to $\mathrm{pH} 5.0$ with $1 \mathrm{M}$-acetic acid and incubated at $37^{\circ} \mathrm{C}$ for $6 \mathrm{~h}$; a third aliquant was incubated similarly but without $\mathrm{pH}$ adjustment. At the end of the incubation, one of the two aliquants at $\mathrm{pH} 5.0$ was re-adjusted back to $\mathrm{pH} 8.0$ with $1 \mathrm{M}-\mathrm{Na}_{2} \mathrm{CO}_{3}$. All treated samples were then boiled for $10 \mathrm{~min}$. Sub-samples were taken for electrophoretic analysis, after which the main samples were dialysed and lyophilized. Bioassays revealed that incubation at $\mathrm{pH} 5.0$ destroyed inhibitory activity, and electrophoretic analysis showed that such treatment also caused a number of protein components to disappear (Fig. 2). No changes were observed following incubation at $\mathrm{pH} 8.0$. It was concluded that an endogenous acid proteinase, only active at acid $\mathrm{pH}$, might be responsible for the destruction of activity.

\section{Effect of inhibitor on chlortetracycline fluorescence patterns in sperm suspensions}

Sperm suspensions were prepared by releasing the epididymal contents of 2 male mice into $2 \mathrm{ml}$ modified Tyrode's medium containing $4 \mathrm{mg}$ BSA (Sigma) per ml (Fraser, 1983a, b). After incubation for $5 \mathrm{~min}$ to allow dispersal, $0.6 \mathrm{ml}$ was removed, mixed with $0.3 \mathrm{ml}$ medium and centrifuged at $11600 \mathrm{~g}_{\max }$ for 4 min to prepare cell-free supernatant containing the inhibitory factor (similar to the crude inhibitor-containing extract described in the 'Materials and Methods'). The remaining suspension was incubated at $37^{\circ} \mathrm{C}$ in $5 \% \mathrm{CO}_{2}-5 \% \mathrm{O}_{2}-90 \% \mathrm{~N}_{2}$ for a further $20 \mathrm{~min}$. Then 2 ali-

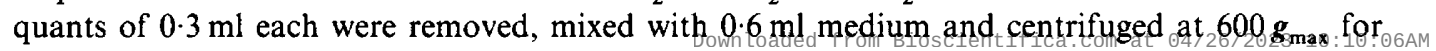




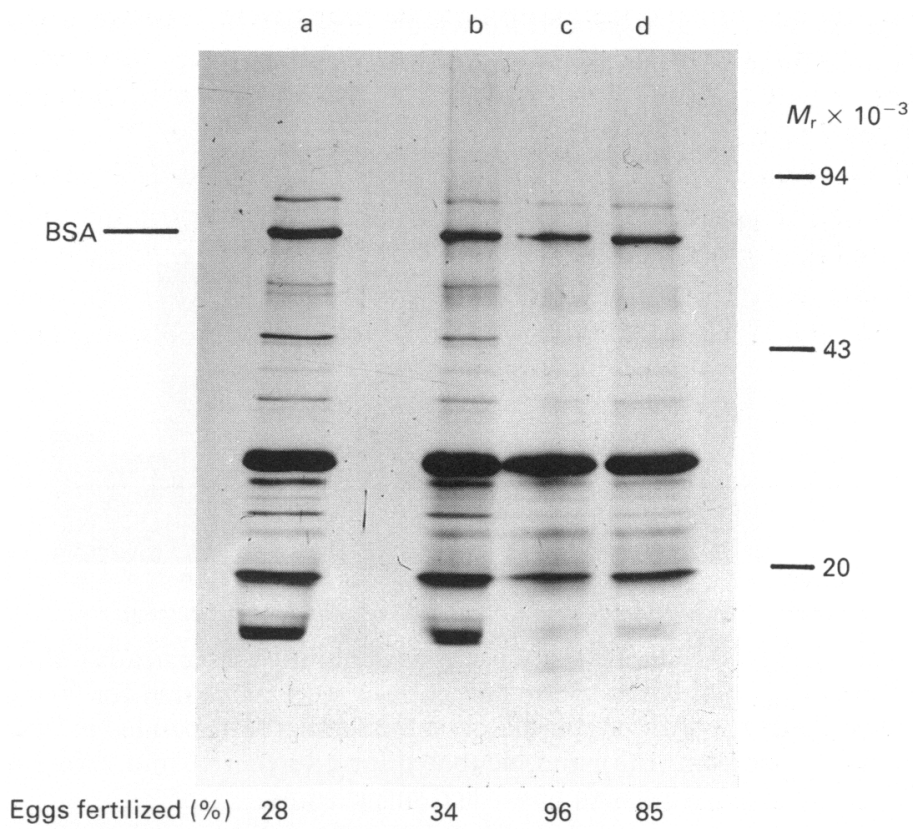

Fig. 2. Changes in partially purified inhibitor preparations during incubation at pH 5.0. Samples were treated as described below and then boiled for $10 \mathrm{~min}$. Sub-samples were taken and later subjected to SDS gel electrophoresis in the reduced state; the rest of each sample was taken for bioassay. The overall percentage of eggs fertilized by each sample is given, below the relevant electropherogram track $(n=2 ;>100$ eggs analysed for each sample). (a) Untreated (i.e. kept at $0^{\circ} \mathrm{C}$ ); this pattern is representative of the partially purified inhibitor preparations (pool B, Fig. 1) used in characterization studies. (b) Incubated for $6 \mathrm{~h}$ at $37^{\circ} \mathrm{C}$ without $\mathrm{pH}$ adjustment (sample in $51 \mathrm{~mm}$-ammonium bicarbonate: approximately $\mathrm{pH} 8 \cdot 0$ ). (c) Incubated for $6 \mathrm{~h}$ at $37^{\circ} \mathrm{C}$ after adjustment to $\mathrm{pH} 5 \cdot 0$. (d) Incubated for $6 \mathrm{~h}$ at $37^{\circ} \mathrm{C}$ after adjustment to $\mathrm{pH} 5 \cdot 0$, then re-adjusted back to $\mathrm{pH} 8 \cdot 0$.

5 min to wash the spermatozoa. Supernatants were removed and pellets were resuspended, one in $0.3 \mathrm{ml}$ fresh medium and one in $0.3 \mathrm{ml}$ inhibitor-containing supernatant. An aliquant from the unwashed suspension served as the control. At $10 \mathrm{~min}$ after resuspension, spermatozoa were stained with CTC to assess their capacitation state (see 'Materials and Methods').

The remaining unwashed suspension was incubated for a further $95 \mathrm{~min}$ (total of $120 \mathrm{~min}$ after release from the epididymides). Two aliquants of $0.2 \mathrm{ml}$ each were removed; one was mixed with $0.2 \mathrm{ml}$ inhibitor-containing supernatant, the other with $0.2 \mathrm{ml}$ fresh medium. After a further $30 \mathrm{~min}$ incubation, both were stained with CTC.

Observed patterns of fluorescence over the sperm heads fell into the three categories F, B, and AR, as described by Ward \& Storey (1984); bright fluorescence was observed in the midpiece region of the tail at all times. Fluorescence patterns in suspensions that had been preincubated for $25 \mathrm{~min}$, washed and then resuspended in inhibitor-containing supernatant did not differ significantly from patterns in unwashed suspensions (Fig. $3 ; n=4$ ); in both, the majority of cells displayed the uncapacitated $\mathrm{F}$ pattern. In contrast, a significantly lower proportion $(P<0.01)$ of the washed cells resuspended in fresh medium displayed the $F$ pattern compared with unwashed cells (means of $28.5 \%$ and $64.0 \%$ respectively); a significantly higher proportion $(P<0.01)$ of the same washed cells displayed the B (capacitated) pattern compared with unwashed cells $(38.5 \%$ and $12.0 \%$ respectively). Although the proportion of AR (acrosome-reacted) cells was higher in the washed suspensions with inhibitor $(33.0 \%)$ than in unwashed suspensions $(24.0 \%$ ) this difference was not 
significant. Washing spermatozoa, a procedure which removes the inhibitor, therefore caused a shift from a predominantly uncapacitated population to a capacitated one.

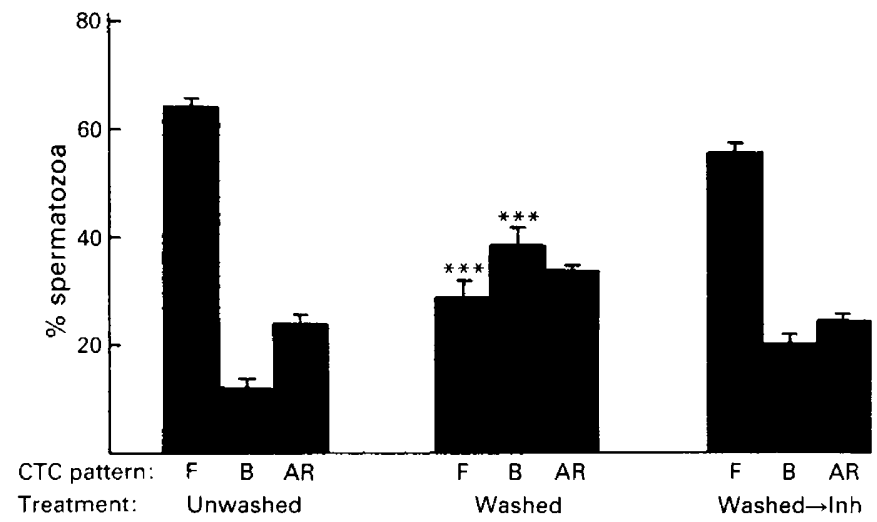

Fig. 3. Chlortetracycline (CTC) fluorescence patterns on mouse sperm heads following removal or addition of inhibitor. Epididymal sperm suspensions were incubated for $30 \mathrm{~min}$ and either washed or left unwashed. The washed spermatozoa were either resuspended in fresh medium or in inhibitor-containing medium (Inh), and incubated for a further $10 \mathrm{~min}$ before being stained with CTC. ${ }^{* * *} P<0.01$ compared with unwashed suspensions.

When sperm suspensions were preincubated for $120 \mathrm{~min}$ and then incubated for a further $30 \mathrm{~min}$ in fresh medium or in inhibitor-containing supernatant, marked differences were again noted between the treatments (Fig. $4 ; n=6)$. Significantly more $(P<0.01)$ of the cells treated with inhibitor-containing medium displayed the $\mathrm{F}$ pattern and significantly fewer displayed the $\mathrm{B}$ $(P<0.05)$ and AR $(P<0.025)$ patterns than did cells incubated with fresh medium. The addition of inhibitor-containing medium to a sperm population, the majority of which were capacitated, therefore inhibited the acrosome reaction and appeared to change the population to one in which the majority of cells with intact acrosomes were uncapacitated.

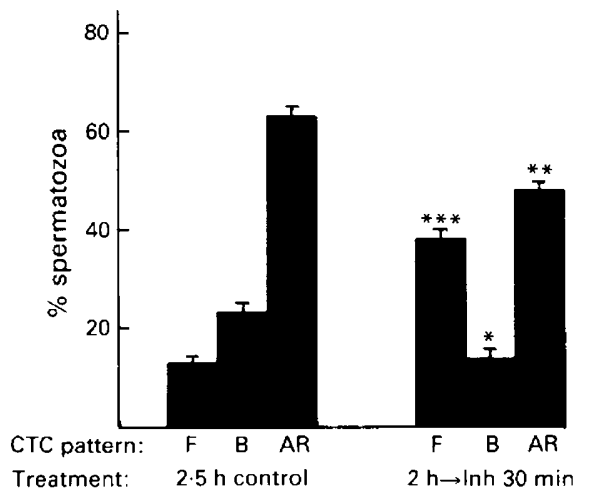

Fig. 4. Chlortetracycline (CTC) fluorescence patterns on mouse sperm heads following addition of inhibitor to capacitated suspensions. Suspensions were incubated for $2 \mathrm{~h}$, then inhibitorcontaining medium (Inh) was added to one aliquant and fresh medium was added to the other. The two aliquants were then incubated for a further $30 \mathrm{~min}$ before being stained with CTC. ${ }^{*} P<0.05,{ }^{* *} P<0.025,{ }^{* * *} P<0.01$ compared with control suspensions.

Evidence that the inhibitor itself was responsible for these changes was obtained by treatment of a capacitated sperm population with fractions obtained from gel filtration of crude inhibitor-

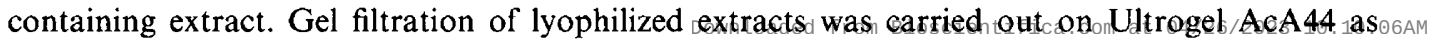


described in the 'Materials and Methods', and the fractions pooled according to fractionation scheme $b$ in Fig. 1 (material eluting before fraction A was also pooled: 'pre-A'). After lyophilization, the pools were coded and randomized. After reconstitution in BSA-supplemented modified Tyrode's medium (described above), the pools were used to dilute sperm suspensions that had been pre-incubated for $120 \mathrm{~min}$ ('capacitated'), in a similar fashion to that described above. Control treatments of dilution in crude inhibitor-containing supernatant and in fresh medium were also included. After a further $30 \mathrm{~min}$ incubation, all suspensions were stained with CTC. The results (Table $7 ; n=2$ ) showed that shifts to an uncapacitated state were only observed with fraction $\mathbf{B}$, the fraction known to contain the inhibitor.

Table 7. Chlortetracycline (CTC) fluorescence pattern of capacitated spermatozoa after treatment with fractions from gel filtration of crude inhibitor

\begin{tabular}{cccc}
\hline & \multicolumn{3}{c}{$\begin{array}{c}\text { CTC fluorescence pattern } \\
\text { (\% of spermatozoa) }\end{array}$} \\
\cline { 2 - 4 } Sample & F & B & AR \\
\hline $\begin{array}{c}\text { Crude inhibitor } \\
\text { (+ ve control) }\end{array}$ & 22.5 & 21.5 & 55.5 \\
Fraction: pre-A & 7 & 19.5 & 73.5 \\
A & 3.5 & 22.5 & 74 \\
B & 21.5 & 26 & 52.5 \\
C & 7.5 & 21.5 & 71 \\
Fresh medium & 7.5 & 19.5 & 73 \\
(-ve control) & & & \\
\hline
\end{tabular}

For nomenclature of fractions, see text and Fig. 1. Values are the means of 2 replicates; each inhibitor sample contained material from $2 \cdot 5$ caudae.

\section{Discussion}

As described in the 'Introduction', a previous study (Fraser, 1984) established that the mouse epididymal factor inhibitory to in-vitro fertilization behaves in the general manner of a decapacitation factor. Removal of inhibitor from uncapacitated sperm suspensions by washing enhances greatly the rapidity with which spermatozoa penetrate eggs, whereas the addition of inhibitor to capacitated suspensions causes fertilization to be delayed. We have now been able to demonstrate that, in parallel with these effects on functional state, the factor has the ability to modify rapidly and reversibly the patterns of CTC fluorescence over mouse sperm heads; this latter is a parameter established by others as reflecting the state of capacitation, at least in mouse (Ward \& Storey, 1984; Neill \& Olds-Clarke, 1987) and possibly in man (Lee et al., 1987). Washing and resuspension of uncapacitated spermatozoa in fresh medium caused a significant shift in the fluorescence from the uncapacitated acrosome-intact $F$ pattern towards the capacitated acrosome-intact $B$ pattern (Fig. 3 ). In contrast, the resuspension of similarly washed, uncapacitated cells in inhibitor-containing medium resulted in a fluorescence pattern which did not differ significantly from that observed in the corresponding unwashed, poorly fertile, spermatozoa; in both suspensions, the majority of cells exhibited the uncapacitated $F$ pattern. The introduction of inhibitor to capacitated suspensions resulted in a shift of fluorescence towards the uncapacitated $F$ pattern, as well as significantly reducing the incidence of acrosome-reacted cells, compared with cells in medium alone $\left(\mathrm{Fig}_{\mathrm{g}} 4\right.$ and 
Table 7). As we have been able to demonstrate that inhibitory activity towards in-vitro fertilization co-migrates on gel filtration with activity towards CTC fluorescence pattern, we believe that the two activities reside in the same macromolecular factor and we therefore consider this factor to be a true decapacitation factor.

Although CTC fluorescence assays allowed direct assessment of capacitation, they were replaced by in-vitro fertilization assays for our studies on isolation and characterization of the decapacitation factor. The in-vitro fertilization assays provided intrinsically more clear-cut results, and required fewer spermatozoa and less fractionated material for each assay, an important consideration in what proved to be very laborious studies. From initial investigations, the inhibitory activity appeared to reside in anionic molecules with approximate $M_{\mathrm{r}} 40000$. However, further attempts to purify the factor or even to correlate activity with an individual protein band detectable on electropherograms failed, and characterization had to be performed on only partially purified preparations. The activity proved stable to heating at $100^{\circ} \mathrm{C}$, and it was not susceptible to hydrolysis by broadspectrum proteinase at a $\mathrm{pH}$ in the range where inhibition in vitro is consistently demonstrable. This was unexpected because, under capacitating conditions, the inhibitory activity is labile; supernatants obtained from sperm suspensions incubated for progressively longer times cause progressively less inhibition (Fraser, 1984). Nevertheless, it is highly likely that the factor contains a polypeptide chain whose secondary structure is required for inhibition, because dithiothreitol treatment in the presence of guanidine hydrochloride destroyed the activity. Despite its apparent stability at $\mathrm{pH} 8$, the factor was susceptible to degradation by endogenous enzymes when the $\mathrm{pH}$ was lowered to 5 . Electrophoresis of material incubated under these conditions provided evidence of proteolytic degradation, although other enzymes (such as glycosidases) could have been involved. As mentioned in the 'Introduction', several decapacitation factors have proved to be glycoproteins, and the stability of the mouse factor to heat and to proteinase action at $\mathrm{pH} 8.0$ might suggest that carbohydrate moieties play an important role in its activity. It was therefore surprising that the factor failed to bind to either concanavalin-A (with high affinity for terminal mannose residues) or wheat-germ agglutinin (with high affinity for $N$-acetylglucosamine residues).

In fact, the mouse decapacitation factor appears to have little in common with the best characterized of the glycoprotein inhibitors. The acrosome-stabilizing factor, purified from rabbit seminal plasma but originating in the epididymis (Thomas et al., 1984), is composed of dissimilar subunits, with mannose and $\mathrm{N}$-acetylglucosamine the primary carbohydrate components; it exists predominantly as a dimer of $M_{\mathrm{r}} 260000$ (Wilson \& Oliphant, 1987). The antifertility factor from human seminal plasma is also a large hetero-dimer of approximate $M_{\mathrm{r}} 200000$ containing mannose and $N$ acetylglucosamine residues; a concanavalin-A column was used during purification of the antifertility factor and detectable activity was confined to material adhering to the column (Audhya et al., 1987). In contrast, the mouse factor did not bind to concanavalin $A$, and there was no evidence for inhibitory activity associated with material larger than BSA $\left(M_{\mathrm{r}} 67000\right)$, e.g. Table 2, fraction (i): no inhibition. Shur \& Hall (1982) have provided evidence that a mouse epididymal glycoprotein containing repeating $N$-acetylglucosamine -galactose residues acts as a decapacitation factor, blocking sperm surface galactosyltransferase acceptor sites needed subsequently for binding to the zona pellucida, and a polylactosamine-containing glycoprotein that inhibits fertilization has been isolated from mouse epididymal fluid by Muller (1988). However, there is no evidence that this polylactosamine material inhibits the acrosome reaction; moreover, it has an apparent $M_{\mathrm{r}}$ of greater than $10^{6}$, and contains lectin-bindable $N$-acetylglucosamine residues (Muller, 1988). By several criteria, therefore, the polylactosamine-based decapacitation factor also differs from that of the mouse.

The mechanism of action of the sperm-associated decapacitation factor remains to be elucidated, a task currently hindered by our lack of knowledge regarding the metabolic changes that engender the state of capacitation. Nevertheless, the fact that egg-associated agonists induce an acrosome reaction only in capacitated cells, apparently via triggering of $\mathrm{Ca}^{2+}$ influx (e.g. Florman et al., 1989), focusses attention on $\mathrm{Ca}^{2+}$ transport. There is indeed evidence that our factor affects $\mathrm{Ca}_{10: 06 \mathrm{AM}}^{2+}$ 
transport, because its removal from sperm suspensions incubated in $\mathrm{Ca}^{2+}$-deficient medium, followed by introduction of millimolar $\mathrm{Ca}^{2+}$, resulted in a significant increase in fertilizing ability when compared with unwashed counterparts receiving such supplementary $\mathrm{Ca}^{2+}$ (Fraser, 1984); in other words, the removal of factor permitted washed $\mathrm{Ca}^{2+}$-deficient cells to respond more quickly to extracellular $\mathrm{Ca}^{2+}$. At least two seminal plasma factors that control $\mathrm{Ca}^{2+}$ transport in spermatozoa have been identified, caltrin in bull (Rufo et al., 1982, 1984) and calsemin in ram and human (Bradley \& Forrester, 1982); however, neither of these has yet been shown to act as a decapacitation factor and in neither case do the reported characteristics match closely those of our mouse factor. Further studies are clearly merited. Our factor's ability to change a population of cells rapidly from the capacitated to the uncapacitated state indicates a critical role in the complex series of events that clearly comprise capacitation. Elucidation of its effect may well help to resolve the details of this latter process.

We thank Andrew Osborne for preparing Figs 3 and 4.

\section{References}

Audhya, T., Reddy, J. \& Zaneveld, L.J.D. (1987) Purification and partial chemical characterization of a glycoprotein with antifertility activity from human seminal plasma. Biol. Reprod. 36, 511-52I.

Austin, C.R. (1951) Observations on the penetration of the sperm into the mammalian egg. Aust. J. sci. Res. $B$ 4, 581-596.

Bedford, J.M. \& Chang, M.C. (1962) Removal of decapacitation factor from seminal plasma by high speed centrifugation. Am. J. Physiol. 202, 179-181.

Bradley, M.P. \& Forrester, I.T. (1982) Human and ram seminal plasma both contain a calcium-dependent regulator protein calsemin. J. Androl. 3, 289-296.

Chang, M.C. (1951) Fertilizing capacity of spermatozoa deposited into the Fallopian tubes. Nature, Lond. 168, 697-698.

Chang, M.C. (1957) A detrimental effect of seminal plasma on the fertilizing capacity of sperm. Nature, Lond. 179, 258-259.

Eng, L.A. \& Oliphant, G. (1978) Rabbit sperm reversible decapacitation by membrane stabilization with a highly purified glycoprotein from seminal plasma. Biol. Reprod. 19, 1083-1094.

Feinberg, J.G. (1976) Modified methods of dialysis. Biochem. J. 153, 721-723.

Florman, H.M., Tombes, R.M., First, N.L. \& Babcock, D.F. (1989) An adhesion-associated agonist from the zona pellucida activates $G$ protein-promoted elevations of internal $\mathrm{Ca}^{2+}$ and $\mathrm{pH}$ that mediate mammalian sperm acrosomal exocytosis. Devl Biol. 135, 133-146.

Fraser, L.R. (1983a) Mouse sperm capacitation assessed by kinetics and morphology of fertilization in vitro. $J$. Reprod. Fert. 69, 419-428.

Fraser, L.R. (1983b) Potassium ions modulate expression of mouse sperm fertilizing ability, acrosome reaction and whiplash motility in vitro. J. Reprod. Fert. 69, 539-553.

Fraser, L.R. (1984) Mouse sperm capacitation in vitro involves loss of a surface-associated inhibitory component. J. Reprod. Fert. 72, 373-384.

Fraser, L.R., Harrison, R.A.P. \& Herod, J.E. (1989) Partial purification and characterization of a decapacitation factor from epididymal mouse spermatozoa. $J$. Reprod. Fert., Abstr. Ser. 3, Abstr. 8.

Harrison, R.A.P. \& Gaunt, S.J. (1988) Multiple forms of ram and bull hyaluronidase revealed by using monoclonal antibodies. J. Reprod. Fert. 82, 777-785.

Lee, M.A., Trucco, G.S., Bechtol, K.B., Wummer, N., Kopf, G.S., Blasco, L. \& Storey, B.T. (1987) Capacitation and acrosome reactions in human spermatozoa monitored by a chlortetracycline fluorescence assay. Fert. Steril. 48, 649-658.

Morrisey, J.H. (1981) Silver stain for proteins in polyacrylamide gels: a modified procedure with enhanced uniform sensitivity. Analyt. Biochem. 117, 307-310.

Muller, C.H. (1988) Mouse sperm fertilizing ability is modified by an epididymal polylactosaminecontaining glycoprotein. Biol. Reprod. 38, Suppl. 1, 143.

Neill, J.M. \& Olds-Clarke, P. (1987) A computerassisted assay from mouse sperm hyperactivation demonstrates that bicarbonate but not bovine serum albumin is required. Gamete Res. 18, 121-140.

Oliphant, G., Reynolds, A.B. \& Thomas, T.S. (1985) Sperm surface components involved in the control of the acrosome reaction. Am. J. Anat. 174, 269-283.

Perham, R.N. (1978) Techniques for determining the amino-acid composition and sequence of proteins. In Techniques in Protein and Enzyme Biochemistry, B110, pp. 1-39. Elsevier/North-Holland Scientific Publishers Ltd, Shannon.

Reddy, J.M., Stark, R.A. \& Zaneveld, L.J.D. (1979) A high molecular weight antifertility factor from human seminal plasma. J. Reprod. Fert. 57, 437-446.

Reddy, J.M., Audhya, T.K., Goodpasture, J.C. \& Zaneveld, L.J.D. (1982) Properties of a highly purified antifertility factor from human seminal plasma. Biol. Reprod. 27, 1076-1083.

Richmond, V.L., St. Denis, R. \& Cohen, E. (1985) Treatment of dialysis membranes for simultaneous dialysis and concentration. Analyt. Biochem. 145, 343-350.

Rufo, G.A., Singh, J.P., Babcock, D.F. \& Lardy, H.A. (1982) Purification and characterization of a calcium transport inhibitor protein from bovine seminal plasma. J. biol. Chem. 257, 4627-4632. 
Rufo, G.A., Schoff, P.K. \& Lardy, H.A. (1984) Regulation of calcium content in bovine spermatozoa. J. biol. Chem. 259, 2547-2552.

Shur, B.D. \& Hall, N.G. (1982) Sperm surface galactosyltransferase activities during in vitro capacitation. $J$. Cell Biol. 95, 567-573.

Snedecor, G.W. \& Cochran, W.G. (1967) Statistical Methods, 6th edn. Iowa State University Press, Ames.

Thomas, T.S., Reynolds, A.B. \& Oliphant, G. (1984) Evaluation of the site of synthesis of rabbit sperm acrosome stabilizing factor using immunocytochemical and metabolic labeling techniques. Biol. Reprod. 30, 693-705.
Ward, C.R. \& Storey, B.T. (1984) Determination of the time course of capacitation in mouse spermatozoa during a chlortetracycline fluorescence assay. Devl Biol. 104, 287-296.

Wilson, W.L. \& Oliphant, G. (1987) Isolation and biochemical characterization of the subunits of the rabbit sperm acrosome stabilizing factor. Biol. Reprod. 37, 159-169.

Yanagimachi, R. (1988) Mammalian fertilization. In The Physiology of Reproduction, pp. 135-185. Eds E. Knobil \& J.D. Neill. Raven Press, New York.

Received 8 August 1989 